\title{
The Effects of Fluid Viscosity on the Orifice Rotameter
}

\author{
Wei-Jiang ${ }^{1}$, Tao-Zhang ${ }^{1}$, Ying-Xu ${ }^{1}$,Huaxiang-Wang ${ }^{1}$,Xiaoli-Guo ${ }^{1}$, Jing-Lei ${ }^{1}$, Peiyong-Sang ${ }^{2}$ \\ ${ }^{I}$ School of Electrical Engineering \& Automation, Tianjin University, Tianjin, 300072, China \\ Tianjin Key Laboratory of Process Measurement and Control, Tianjin, 300072, China \\ ${ }^{2}$ Flow Measurement Center of Aviation Industries of China,Xinxiang,453049, China \\ Corresponding author: Ying-Xu E-mail: xuying @ tju.edu.cn
}

\begin{abstract}
Due to the viscous shear stress, there is an obvious error between the real flow rate and the rotameter indication for measuring viscous fluid medium. At $50 \mathrm{cSt}$ the maximum error of DN40 orifice rotameter is up to $35 \%$. The fluid viscosity effects on the orifice rotameter are investigated using experimental and theoretical models. Wall jet and concentric annulus laminar theories were adapted to study the influence of viscosity. And a new formula is obtained for calculating the flow rate of viscous fluid. The experimental data were analyzed and compared with the calculated results. At high viscosity the maximum theoretical results error is $6.3 \%$, indicating that the proposed measurement model has very good applicability.
\end{abstract}

Keywords: Rotameter, viscosity, experiment, regional model, flow measure.

\section{INTRODUCTION}

In a typical variable area flow meter [1], the orifice rotameter has the advantages of simple structure, high reliability, convenient maintenance, low pressure loss, etc. and is widely used in industrial flow measurement. When measuring a viscous fluid such as fossil oil, lube, beverages, and dairy products, an obvious indication error OCCURS due to the viscous shear stress effect. At $50 \mathrm{cSt}$ the maximum DN40 orifice rotameter error can be up to $35 \%$. It is therefore important and practical to study the fluid viscosity effects on the orifice rotameter.

Early research focused mainly on the rotameter measurement principle. Schoenborn and Colburn [2] first began to deduce the rotameter flow equation. They thought that the rotameter could be regarded as a variable crosssection orifice flow meter. According to this analogy the relationship between the flow rate and the differential pressure was directly obtained. The rotameter differential pressure was derived based on the Bernoulli equation. A rotameter flow equation with the same Schoenborn form was then obtained by Whitwell, Plumb, and Polentz [3], [4] through solving the continuity equation with differential pressure.

Modern research is concentrated mainly on two aspects, first is the low rotameter accuracy in industrial applications. In order to improve the rotameter measurement precision and expand the measurement range, the rotameter structure was optimized by redesigning the float shape and strictly controlling the production process. A cone float was designed and the designed rotameter flow equation was derived by Urata [5]. Through experiments he found that the outflow coefficient of the newly designed rotameter fell within a wide range of Reynolds numbers (minimum to 50) that remained constant, showing that viscosity has less influence on this rotameter. Vallascas [6] developed the magnetic suspension rotameter. An electric solenoid was installed in the outer conical tube to keep the float in a fixed position. The magnetic force was generated by the interaction of the variable magnetic field between the float magnetism and the electric solenoid current. The current output is proportional to the volume flow. Liu et al. [7] developed a capacitance sensor for the rotameter. The simulation and practical flow experiments were carried out with air as the medium. Baker [8], [9] researched the influence of each production process on the rotameter measurement performance. Sondh et al. [10], [11] designed 3 different float shapes: a cone frustum, cone frustum with hemispherical base, and cone frustum with hemispherical base and parabolic apex. A compensation algorithm based on the BP neural network by Ning et al. [12] was used to eliminate the temperature drift in metal tube rotameter using an off-line training method based on the virtual instrument.

The computational fluid dynamics (CFD) method can be used to calculate the pressure, velocity distribution and other aspects of the data in the flow field, and also design the product structural parameters. The German scholars Bueckle and Durst [13], [14] first introduced the CFD method into rotameter research and exploited the Laser Doppler 
Anemometer (LDA) to verify the results. It was concluded that the simulation results were consistent with the LDA test results. The reasons for the differences between the numerical and experimental data were analyzed. Their research showed that the CFD method could analyze the rotameter internal flow field accurately and efficiently.

As seen from the above, in the structural optimization aspect, researchers mainly adopted the CFD method to optimize the float structure. The optimized results were verified experimentally.

The other aspect is the fluid viscosity effect on measurement precision. A lot of research work has been done [15], [16], with several viscosity correction schemes put forward in this aspect. Fisher [17] first proposed a design that ignored the viscous effects, but the weight of the designed float was too small, and could not be applied to practical production. A series of special float shapes that did not need to correct under certain viscosities were designed by Miller [18].

From the data available for small rotameters that use spherical floats in gas flow, Levin and Escorza [19] found a linear relationship for variable volumetric flow $Q$, density $\rho$, and viscosity $\mu$ at a constant float height. At low Reynolds numbers $(\operatorname{Re}<1), Q \mu$ became a constant; while at high Reynolds numbers $(\operatorname{Re}>2000), \mathrm{Q} \mu^{1 / 2}$ became a constant. This method can be used to calibrate a gas rotameter indirectly after the density and viscosity of the fluid has been determined.

Assuming that flow coefficient in the flow equation was a simple function of the Reynolds number and the viscosity coefficient was replaced by the Reynolds number to characterize the viscosity change, Wojtkowiak [20] obtained a nonlinear equation for the flow rate with the float heights through rotameter flow experimental data at different float heights. The viscosity flow to theoretical flow ratio and the viscosity correction curve were then obtained.

The study of viscosity effects on rotameter measurement can be divided into two categories. The first category is based on the existing float type flowmeter. The viscosity correction curve is determined through experimental and theoretical analysis, and thus the flow conversion relationship between different viscosities is obtained. The second category is based on the float structural design. The design is verified and improved through experimentation to eliminate the viscosity effect.

The simulation or experimental method is generally adopted to optimize the float design and correct the float viscosity. Analysis and research into the flow field distribution and interaction between the fluid and float is lacking. The correction curve can be suitable only for a specific caliber. Rotameters with different calibers need to fit many different curves, so this method lacks universality. The optimal design depends on the designer's experience and the cost of experimental verification is high with a long cycle. The concentric annulus laminar flow and wall jet theory were therefore adopted to study the flow field distribution and the float viscous shear stress. The flow field was divided into three regions according to the relative position of the float and the orifice. Viscous friction and pressure drop were calculated respectively in each region to accurately analyze the viscous friction and pressure difference, determine the flow field distribution and analyze the fluid viscosity effects on the rotameter.

\section{MODELING WITH WALL JET AND CONCENTRIC ANNULUS LAMINAR THEORY}

Viscous fluid flows from the bottom to the top of the float. Acting on the float force is the pressure drag $F_{p}$, buoyancy $F_{\rho}$, gravity $G$, and the viscous friction $F_{\tau}$, respectively.

The force balance equation:

$$
F_{P}+F_{\tau}=G-F_{\rho}
$$

In the above formula, $G=\rho_{f l} V_{f l} g, F_{\rho}=\rho V_{f l} g, \rho_{f l}$ is the density of the float, $V_{f l}$ is the volume of the float, $\rho$ is the density of the fluid, and calculated directly. Differential pressure and viscous friction can be calculated indirectly. As shown in Fig.1. to calculate the differential pressure and viscous friction accurately, the flow field is divided into 3 regions. Region $\mathrm{A}$ is the area from the bottom of the float to the bottom of the orifice. The flow field of region $\mathrm{A}$ is influenced mainly by the tube wall and the float, less affected by the orifice plate. Region B is the zone between the orifice plate and float, which has the maximum velocity and maximum pressure gradient in the whole flow field. Region $\mathrm{C}$ is the flow area through the orifice, which is also the confined annular wall jet region.

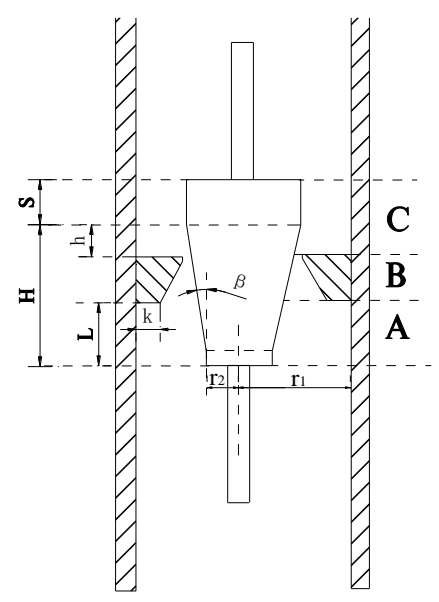

Fig.1. The orifice rotameter.

\subsection{Calculation of region $A$}

As shown in Fig.1. $r_{2}$ is the float bottom radius, $r_{1}$ is the tube radius. The float structure has axial symmetry; the flow is axisymmetric, so $\frac{\partial}{\partial \theta}=0$. The main flow direction is $\mathrm{z}$. The flow is steady flow, so $\frac{\partial u}{\partial t}=0$.

The Laminar concentric annulus solution is [21]

$$
u=-\frac{1}{4 \mu} \frac{d p}{d z}\left(r_{1}^{2}-r^{2}+\frac{r_{1}^{2}-r(z)^{2}}{\ln \left(r_{1} / r(z)\right)} \ln \left(\frac{r}{r_{1}}\right)\right)
$$




$$
Q=\int_{r(z)}^{r_{1}} 2 \pi r u d r=\frac{\pi}{8 \mu}\left[\left(r_{1}^{4}-r(z)^{4}\right)-\frac{\left(r_{1}^{2}-r(z)^{2}\right)^{2}}{\ln \left(r_{1} / r(z)\right)}\right] \frac{d p}{d z}
$$

$r(z)=r_{2}+z \tan \beta . \beta$ is the float cone angle.

The pressure drops in region A are calculated first.

$$
\begin{gathered}
\frac{d p}{d z}=\frac{8 \mu Q}{\pi\left[\left(r_{1}^{4}-r(z)^{4}\right)-\frac{\left(r_{1}^{2}-r(z)^{2}\right)^{2}}{\ln \left(r_{1} / r(z)\right)}\right]} \\
\ln \frac{r_{1}}{r(z)}=2\left\{\frac{\frac{r_{1}}{r(z)}-1}{\frac{r_{1}}{r(z)}+1}+\frac{1}{3}\left(\frac{\frac{r_{1}}{r(z)}-1}{\frac{r_{1}}{r(z)}+1}\right)^{3}+\frac{1}{5}\left(\frac{\frac{r_{1}}{r(z)}-1}{\frac{r_{1}}{r(z)}+1}\right)^{5}+\cdots\right\} \approx \frac{2\left(r_{1}-r(z)\right)}{\left(r_{1}+r(z)\right)}
\end{gathered}
$$

Substitute (5) into (4)

$$
d p=\frac{16 \mu Q}{\pi\left(r_{1}+r(z)\right)\left(r_{1}-r(z)\right)^{3}} d z
$$

Set $\mathrm{L}$ as the length of float in region $\mathrm{A}$, so there is

$$
\begin{aligned}
\Delta p_{A}= & \int_{0}^{L} \frac{16 \mu Q}{\pi\left(r_{1}+r(z)\right)\left(r_{1}-r(z)\right)^{3}} d z \\
= & \frac{4 \mu Q}{\pi r_{1} \tan \beta}\left[\frac{1}{2 r_{1}^{2}} \ln \left(\frac{r_{1}+r_{2}+L \tan \beta}{r_{1}+r_{2}} \frac{r_{1}-r_{2}}{r_{1}-\left(r_{2}+L \tan \beta\right)}\right)+\frac{L \tan \beta\left(2 r_{1}-2 r_{2}-L \tan \beta\right)}{\left(r_{1}-\left(r_{2}+L \tan \beta\right)\right)^{2}\left(r_{1}-r_{2}\right)^{2}}\right. \\
& \left.+\frac{L \tan \beta}{\left(r_{1}-\left(r_{2}+L \tan \beta\right)\right)\left(r_{1}-r_{2}\right) r_{1}}\right]
\end{aligned}
$$

The viscous friction on the float is then calculated in region $\mathrm{A}$, differentiate (2)

$$
\frac{\partial u}{\partial r}=-\frac{r}{2 \mu} \frac{d p}{d z}+\frac{1}{r} \frac{d p}{d z} \frac{1}{4 \mu} \frac{r_{1}^{2}-r(z)^{2}}{\ln \left(r_{1} / r(z)\right.}
$$

The frictional resistance is

$$
\tau=\mu \frac{\partial u}{\partial r}=\frac{d p}{d z}\left[\frac{\left(r_{1}+r(z)\right)^{2}}{8 r(z)}-\frac{r(z)}{2}\right]
$$

Substitute (4) into 9),

$$
F_{\tau}=2 \pi \int_{0}^{L} \frac{16 \mu Q r(z)}{\pi\left(r_{1}+r(z)\right)\left(r_{1}-r(z)\right)^{3}}\left[\frac{\left(r_{1}+r(z)\right)^{2}}{8 r(z)}-\frac{r(z)}{2}\right] d z
$$

Simplification of the result is

$$
F_{\tau A}=\frac{2 \mu Q}{\tan \beta}\left[\frac{4 L \tan \beta}{\left(r_{1}-r_{2}\right)\left(r_{1}-\left(r_{2}+L \tan \beta\right)\right)}-\frac{1}{r_{1}} \ln \left(\frac{r_{1}^{2}-\left(r_{2}+L \tan \beta\right)^{2}}{r_{1}^{2}-r_{2}^{2}}\right)\right]
$$

This is the float fluid friction in region A. The direction is along the float wall.

\subsection{Calculation of region $B$}

As shown in Fig.2. a front step exists between region A and $\mathrm{B}$, the radial height is $\mathrm{k}$. The pressure drop between region $\mathrm{A}$ and $\mathrm{B}$ can be directly obtained using the Bernoulli equation,

$$
\Delta p_{A B}=\frac{\rho Q^{2}}{2}\left(\frac{1}{S_{B}^{2}}-\frac{1}{S_{A}^{2}}\right)
$$

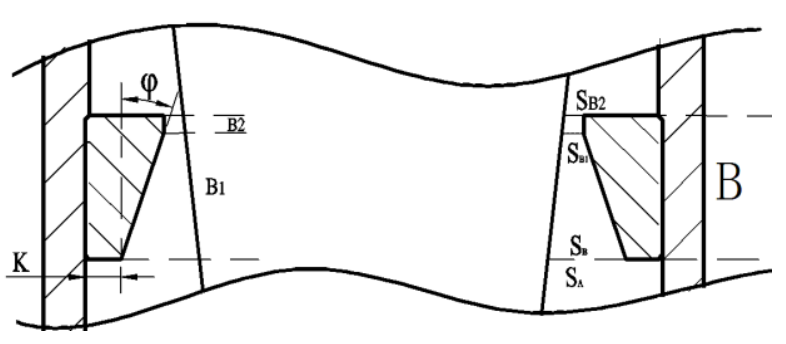

Fig.2. Region B.

Pressure drop and friction in region $\mathrm{B}$ are calculated as follows. Region B could be divided into 2 areas as shown in Fig.3. The pipe walls of region $\mathrm{B}_{2}$ do not vary with the change of $z$. The surfaces of the pipe in region $B_{1}$ changes with the $\mathrm{z}$ coordinate. The change rule is

$$
r_{1}^{\prime}=r_{1}-k-(z-L) \tan \varphi
$$

Compared with region $\mathrm{A}$, there is just tube wall surface change at $\mathrm{z}$ direction in region $\mathrm{B}_{1}$, but the friction and pressure drop form do not change. Equation (13) is substituted into (7).

$$
\begin{aligned}
\Delta p_{B_{1}} & =\int_{L}^{H-h} \frac{16 \mu Q}{\pi\left[r_{1}^{\prime}+r(z)\right]\left[r_{1}^{\prime}-r(z)\right]^{3}} d z \\
& =\frac{16 \mu Q}{\pi} \int_{L}^{H-h} \frac{1}{\left(J_{1}+z J_{3}\right)\left(J_{2}+z J_{4}\right)^{3}} d z
\end{aligned}
$$

Here $\mathrm{h}$ is the float displacement in the current flow. $\mathrm{H}$ is the length from float bottom to the top of the flow bevel, as shown in Fig.1. set

$$
\begin{gathered}
J_{1}=r_{1}+r_{2}-k+L \tan \varphi \\
J_{2}=r_{1}-r_{2}-k+L \tan \varphi \\
J_{3}=\tan \beta-\tan \varphi \\
J_{4}=-\tan \beta-\tan \varphi
\end{gathered}
$$

Through (14) the pressure drops in region B could be calculated as

$$
\begin{aligned}
\Delta p_{B_{1}} & =\frac{16 \mu Q}{\pi} \int_{L}^{H-h} \frac{1}{\left(J_{1}+z J_{3}\right)\left(J_{2}+z J_{4}\right)^{3}} d z \\
& =\frac{16 \mu Q}{\pi}\left[I_{1}(H-h)-I_{1}(L)\right]
\end{aligned}
$$

Function $I_{1}(z)$ is defined as

$$
I_{1}(z)=\frac{J_{3}{ }^{2} \ln \frac{J_{2}+z J_{4}}{J_{1}+z J_{3}}}{\left(J_{1} J_{4}-J_{2} J_{3}\right)^{3}}+\frac{J_{3}}{\left(J_{1} J_{4}-J_{2} J_{3}\right)^{2}\left(J_{2}+z J_{4}\right)}-\frac{1}{2\left(J_{1} J_{4}-J_{2} J_{3}\right)\left(J_{2}+z J_{4}\right)^{2}}
$$


Also the friction of $\mathrm{B}_{1}$ area could be obtained by substituting (13), (15) (18) into (10)

$$
F_{\tau B_{1}}=\mu Q\left[I_{2}(H-h)-I_{2}(L)\right]
$$

Function $\mathrm{I}_{2}(\mathrm{z})$ is defined as

$$
I_{2}(z)=\frac{2}{J_{1} J_{4}-J_{2} J_{3}} \ln \frac{J_{2}+z J_{4}}{J_{1}+z J_{3}}-\frac{4}{J_{4}\left(J_{2}+z J_{4}\right)}
$$

The pressure drop in $\mathrm{B}_{2}$ is calculated by the Bernoulli equation, as follows

$$
\Delta p_{B_{2}}=\frac{\rho Q^{2}}{2}\left(\frac{1}{S_{B_{2}}^{2}}-\frac{1}{S_{B_{1}}^{2}}\right)
$$

As shown in Fig.2. $\mathrm{S}_{\mathrm{B} 1}$ is the annulus area at the junction of $\mathrm{B}_{1}$ and $\mathrm{B}_{2} . \mathrm{S}_{\mathrm{B} 2}$ is the annulus area at the outlet of the wall jet. The friction in $\mathrm{B}_{2}$ is calculated with the friction in region C. The reason will be presented in the following section.

\subsection{Calculation of region $C$}

As fluid flows through the gap between the orifice plate and the float into the area of $\mathrm{C}$, the annular wall jet is formed as shown by the dotted lines in Fig.3. with the diffusion angle $\alpha$ of wall jet [22]

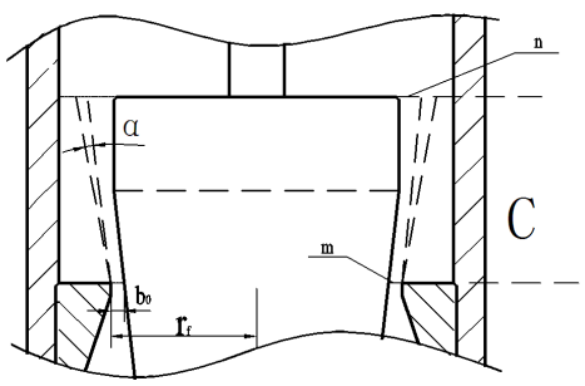

Fig.3. The wall jet in region $\mathrm{C}$.

In Fig.3. the lateral dotted line is formed by the points whose velocities are zeros in the $\mathrm{z}$ direction, and could be regarded as a wall in the calculation of pressure drop. The flow model in region $\mathrm{C}$ could be simplified as the concentric annular diffusion flow as shown in Fig. 4.

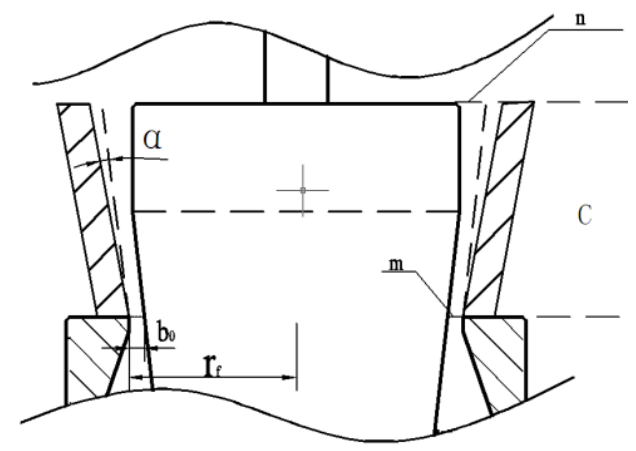

Fig.4. Simplified model.
The calculation method is similar to region B. Pressure drop in region $\mathrm{C}$ is

$$
\Delta p_{C}=\frac{16 \mu Q}{\pi} \int_{H-h}^{H+S} \frac{1}{\left(c_{1}+z c_{3}\right)\left(c_{2}+z c_{4}\right)^{3}} d z
$$

Here

$$
\begin{gathered}
c_{1}=2 r_{2}+b_{0}+(-H+h)[\tan (\beta+\alpha)-\tan \beta] \\
c_{2}=b_{0}+(-H+h)[\tan (\beta+\alpha)-\tan \beta] \\
c_{3}=\tan (\beta+\alpha)+\tan \beta \\
c_{4}=\tan (\beta+\alpha)-\tan \beta
\end{gathered}
$$

$b_{0}=r_{f}-r_{2}-(H-h) \tan \beta$, is the width of the jet outlet. There is

$$
\begin{aligned}
\Delta p_{C} & =\frac{16 \mu Q}{\pi} \int_{H-h}^{H+S} \frac{1}{\left(c_{1}+z c_{2}\right)\left(c_{3}+z c_{4}\right)^{3}} d z \\
& =\frac{16 \mu Q}{\pi}\left[I_{1}(H+S)-I_{1}(H-h)\right]
\end{aligned}
$$

$\mathrm{I}_{1}(\mathrm{z})$ function is defined by (20).

The friction force of region $\mathrm{C}$ cannot be calculated using the divergent tube model. This is because the flow field in region $\mathrm{C}$ is limited by the wall jet, the maximum velocity of the profile $u_{\mathrm{m}}$ is closer to the wall compared with the diverging tube and the viscous friction force on the float is larger.

According to the wall jet theory the maximum velocity of the profile $u_{\mathrm{m}}$ and the outlet velocity $u_{0}$ have the following relationship [23],

$$
\frac{u_{m}}{u_{0}}=3.50 \sqrt{\frac{b_{0}}{z}}
$$

As shown in Fig.4. the ratio of the velocity at separation point $\mathrm{n}$ of float boundary layer to the outlet $\mathrm{m}$ is calculated according to (30), and the results are shown in the following table.

Table 1. Ratios of the velocity at $\mathrm{n}$ to outlet velocity at $\mathrm{m}$.

\begin{tabular}{cccc}
\hline $\mathrm{h}$ & $\mathrm{b}_{0}$ & $(\mathrm{~h}+\mathrm{S}) / \mathrm{b}_{0}$ & $\mathrm{u}_{\mathrm{m}} / \mathrm{u}_{0}$ \\
\hline 0.0049 & 0.000738 & 22.9 & 0.73 \\
0.0123 & 0.001471 & 16.52 & 0.86 \\
0.0192 & 0.002154 & 14.49 & 0.92 \\
0.0284 & 0.003065 & 13.18 & 0.96 \\
0.0362 & 0.003837 & 12.56 & 0.99 \\
\hline
\end{tabular}

The table shows that even at the farthest point away from the jet outlet, the reduction of maximum velocity is very small. The flow field in region $\mathrm{C}$ is confined by the wall jet, 
so the diffusion angle is less than the wall jet diffusion angle [24], and the maximum velocity profile is larger than that of the wall jet. It can be approximately considered that the viscous friction on the float in region $\mathrm{C}$ is everywhere equal, and equal to outlet friction. The total friction of region $\mathrm{C}$ and $\mathrm{B}_{2}$ can be concluded.

$$
F_{\tau, C+B_{2}}=\frac{4 \mu Q(h+S)\left\{r_{f}+3\left[r_{2}+(H-h) \tan \beta\right]\right\}}{\left[r_{f}+r_{2}+(H-h) \tan \beta\right]\left[r_{f}-r_{2}-(H-h) \tan \beta\right]^{2}}
$$

\subsection{Diffusion angle of the confined wall jet}

As shown in Fig.6. the fluid jets from the nozzle outlet. The entrainment of ambient fluid is produced in the upper boundary as a free jet and this mixing force develops downwards. The boundary layer is formed in the lower boundary by the solid wall friction effect and develops upward with a potential core area in the center. According to the maximum velocity of profile $\mathrm{u}_{\mathrm{m}}$, the flow field is divided into two areas: the upper mixing zone and the lower boundary layer [25].

Verhoff [26] and Rajaratnam [27] presented the empirical formula of the relationship between the maximum profile velocity and the outlet velocity. Rajaratnam also studied the entrainment velocity in the free mixing zone and the effects of the surface roughness on the thickness of the boundary layer.

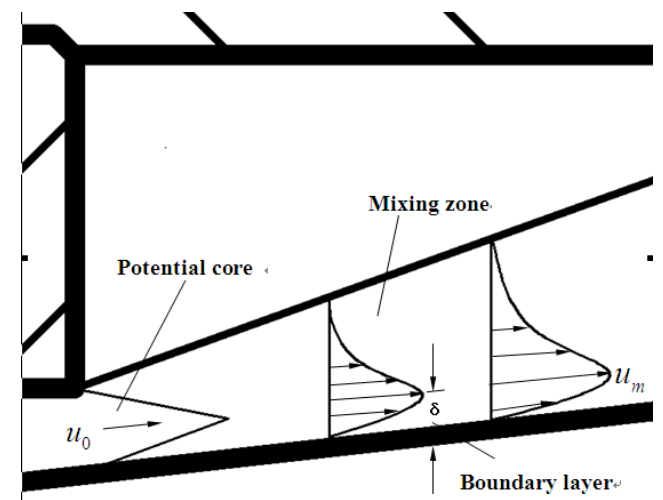

Fig.5. The confined wall jet.

The diffusion angle does not change with the variance in the outlet velocity [28]. The diffusion angle is therefore determined by the outlet width and the cross-sectional area of the concentric annular pipe after the outlet. Therefore the dimensionless coefficient $\mathrm{K}$ is defined to characterize the degree of limitation. [29]

$$
K=\frac{b_{0}}{\sqrt{A_{1}}}
$$

The diffusion angle of the wall jet is determined using simulation (CFD). The relationship between the diffusion angle $\alpha$ and $\mathrm{K}$ is then obtained.

$$
\alpha=11.543-43.998 \mathrm{~K}
$$

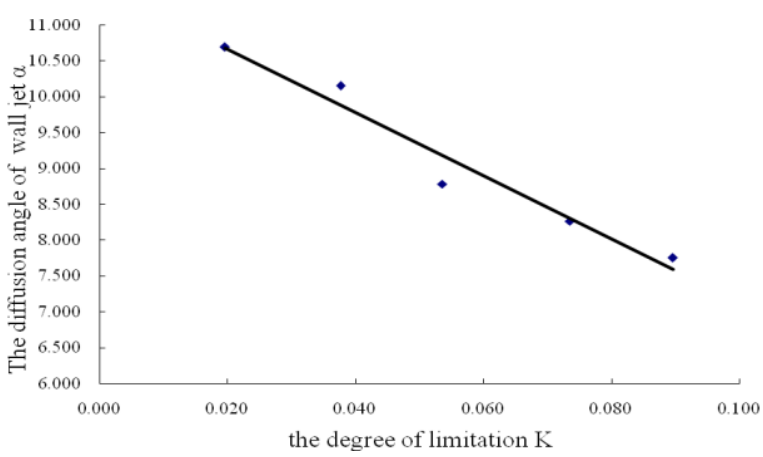

Fig.6. The relationship of K and the diffusion angle $\alpha$.

Equation 33 illustrates that the diffusion angle is inversely proportional to the float limitation to the flow field.

\subsection{Theoretical flow rate}

According to the above derivation process, the friction and pressure drop in each region are obtained, so,

$$
\begin{gathered}
F_{\tau}=F_{\tau A}+F_{\tau B_{1}}+F_{\tau, B_{2}+C} \\
\Delta P=\Delta P_{A}+\Delta P_{A B}+\Delta P_{B_{1}}+\Delta P_{B_{2}}+\Delta P_{C}
\end{gathered}
$$

$F_{\tau A}, \quad F_{\tau B_{1}}, \quad F_{\tau, B_{2}+C}$ are decided by (11), (21), (31), respectively, and are the linear function of the flow rate $\mathrm{Q}$;

$\Delta P_{A}, \Delta P_{B_{1}}, \Delta P_{C}$ are dominated by (7), (19), (29), respectively, and are the linear function of $\mathrm{Q}$;

$\Delta P_{A B}, \Delta P_{B_{2}}$ are ruled by (12), (23), respectively, and are the quadratic function of $\mathrm{Q}$.

Substitute (34), (35) into the formula (1)

$$
F_{\tau}+A_{f} \Delta P=G-F_{\rho}
$$

Quadratic equation of flow rate $\mathrm{Q}$ is obtained.

$$
a_{1} Q^{2}+a_{2} Q=a_{3}
$$

$a_{1}, a_{2}, a_{3}$ are the coefficient of equation.

$$
\begin{aligned}
a_{1} Q^{2} & =A_{f}\left(\Delta P_{A B}+\Delta P_{B_{2}}\right) \\
& =A_{f}\left[\frac{\rho Q^{2}}{2}\left(\frac{1}{S_{B}^{2}}-\frac{1}{S_{A}^{2}}\right)+\frac{\rho Q^{2}}{2}\left(\frac{1}{S_{B_{1}}^{2}}-\frac{1}{S_{B_{2}}^{2}}\right)\right] \\
& a_{1}=\frac{A_{f} \rho}{2}\left(\frac{1}{S_{B}^{2}}-\frac{1}{S_{A}^{2}}+\frac{1}{S_{B_{1}}^{2}}-\frac{1}{S_{B_{2}}^{2}}\right)
\end{aligned}
$$

Similarly

$$
a_{2} Q=F_{\tau A}+F_{\tau B_{1}}+F_{\tau, B_{2}+C}+A_{f}\left(\Delta P_{A}+\Delta P_{B_{1}}+\Delta P_{C}\right)
$$




$$
a_{3}=G-F_{\rho} \text {. }
$$

Finally the theoretical flow rate Q is obtained.

$$
Q=\frac{-a_{2}+\sqrt{a_{2}^{2}+4 a_{1} a_{3}}}{2 a_{1}}
$$

\section{EXPERIMENTAL RESEARCHES}

\subsection{The experimental water flow device}

The experimental water flow device at Tianjin University Flow Laboratory was used to perform the calibration, as shown in Fig.7. Water flow was applied to regulate the pressure and the device flow rate was continuously adjusted. Both the weighting and master meter methods were used with the experimental device for verification. The weighing method uncertainty is $0.05 \%$, and that for the master meter method is $0.2 \%$.

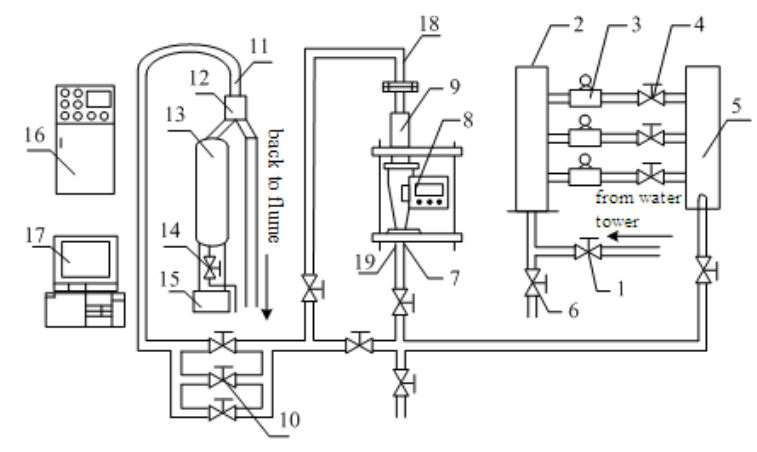

Fig.7. Standard water device.

1. Inlet Valve, 2. Filter Tank, 3. Master Meter, 4. Electric Control Valve, 5. Surge Tank, 6. Excluding-ordure Valve, 7. Support Plate, 8. Metal Tube Rotameter, 9. Clamping, 10. Flow Regulating Valve, 11. Nozzle, 12. Commutator, 13. Counter Tank, 14. Drain Valve, 15. Electronic Weigher, 16. Control Cabinet, 17. Computer 18,19 . pressure measuring hole.

$q_{\mathrm{v} 0 \min }, 0.25 q_{\mathrm{v} 0 \max }, 0.4 q_{\mathrm{v} 0 \max }, 0.7 q_{\mathrm{v} 0 \max }$ and $q_{\mathrm{v} 0 \max }$ are selected, and weighting method is used to calibrate DN50 metal tube rotameter. Measuring range is $1 \sim 10 \mathrm{~m}^{3} / \mathrm{h}$ on the standard device.

Table 2. The water flow device verification data.

\begin{tabular}{cccc}
\hline $\mathrm{h}(\mathrm{mm})$ & $\begin{array}{c}\text { the Indicating } \\
\text { value }\left(\mathrm{m}^{3} / \mathrm{h}\right)\end{array}$ & $\begin{array}{r}\text { Experimental } \\
\text { flow rate }\left(\mathrm{m}^{3} / \mathrm{h}\right)\end{array}$ & $\begin{array}{l}\text { Error } \\
(\%)\end{array}$ \\
\hline 4.90 & 1.00 & 1.01 & 1.20 \\
13.20 & 2.50 & 2.51 & 0.24 \\
19.40 & 4.00 & 3.98 & -0.60 \\
28.90 & 7.00 & 7.07 & 0.97 \\
36.50 & 10.00 & 9.96 & -0.36 \\
\hline
\end{tabular}

Data in Table 2. showed that the uncertainty of the rotameter reached 1.5 grade standard.

\subsection{Experiments with viscous fuild}

4050 aviation lubricant oil was used as the experimental medium. The viscosity range for this oil is from $10 \mathrm{cSt}$ to $50 \mathrm{cSt}$. The device is composed of seven parts: a liquid circulation system, test tube, static weighing system, startstop equipment, electric heater, refrigeration unit and the control system. Weighing method was used to calibrate the rotameter. The valve was opened and the oil in the tank pumped out using the oil pump (Group). After the oil was filtered the temperature was changed (heating or cooling), the pressure stabilized and the oil circulated into the oil tank through the commutator's bypass pipeline. When the flow rate and oil temperature met the requirements, the standard scale was reset and the initial readings noted $m_{0}(\mathrm{~kg})$. The commutator was started, allowing the oil to flow into the weighing container. This simultaneously triggered the timer and started the count. When the desired weighting value was achieved, the commutator was started again so that the oil could flow through the bypass line back into the tank. The timer was stopped when the oil was back inside the tank. The standard scale $m(\mathrm{~kg})$ and time $t$ (s) values were recorded. The average flow of $q_{m}$ through the rotameter can be calculated using the following formula,

$$
q_{m}=\frac{m-m_{0}}{t} \varepsilon
$$

here

$\varepsilon=\frac{\rho_{w}}{\rho_{w}-\rho_{a}}-$ The correction coefficient for air buoyancy

$$
\begin{aligned}
& \rho_{a}-\text { The density of air, } \mathrm{kg} / \mathrm{m}^{3} \\
& \rho_{w}-\text { The density of oil, } \mathrm{kg} / \mathrm{m}^{3}
\end{aligned}
$$

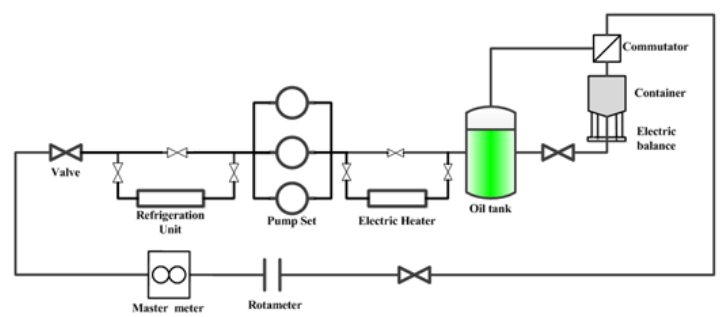

Fig.8. Principle of the variable viscosity device.

The experimental device performances index is as follows: temperature change range from $-35^{\circ} \mathrm{C}$ to $150^{\circ} \mathrm{C}$, temperature control accuracy within $1^{\circ} \mathrm{C}$, flow range from $0.1 \mathrm{~m}^{3} / \mathrm{h}$ to $80 \mathrm{~m}^{3} / \mathrm{h}$, and expanded uncertainty $(\mathrm{K}=2)$ is about $0.05 \%$.

To avoid errors caused by float shake and parallax errors in the calibration process, $4 \sim 20 \mathrm{~mA}$ output analog signals were converted and collected in the corresponding sampling time for calculating the average flow rate.

The experimental procedure was as follows:

1) The flow meter was calibrated in the variable viscosity flow device. Each flow point was calibrated twice at positive and reverse range. The calibration process was as follows: first adjust the frequency transformer to make the 
measured voltage value corresponding to the indication flow rate of $q_{\mathrm{v} 0 \min }, 0.25 q_{\mathrm{v} 0 \max }, 0.4 q_{\mathrm{v} 0 \max }, 0.7 q_{\mathrm{v} 0 \max }$ and $q_{\mathrm{v} 0 \max }$, respectively. The medium and flow rate temperatures were recorded to calculate the average positive and reverse range values.

2) The lubricating oil was taken from the variable viscosity experimental system. The sample temperature was kept the same as the lubricating oil temperature in the device. The lubricating oil viscosity was measured using a NDJ-5S digital viscometer and the density was measured using a densimeter.

3) The medium temperature was changed to change the medium viscosity and density. Steps (1) (2) were then repeated.

\subsection{Experiment data and analysis}

The experimental data are shown in Table 3. - Table 6.

Table 3. The experimental data for $v=9.99 \mathrm{cSt}$.

\begin{tabular}{rrrrrr}
\hline $\mathrm{q}_{\mathrm{v} 0} / \mathrm{m}^{3} / \mathrm{h}$ & $\mathrm{t} /{ }^{\circ} \mathrm{C}$ & $\rho / \mathrm{kg} / \mathrm{m}^{3}$ & $\mathrm{v} / \mathrm{cSt}$ & $\mathrm{q}_{\mathrm{v}} / \mathrm{m}^{3} / \mathrm{h}$ & $\delta_{1} / \%$ \\
\hline 1.03 & 67.10 & 970.12 & 10.23 & 0.97 & 0.52 \\
2.65 & 66.78 & 970.11 & 10.33 & 2.46 & 1.95 \\
3.95 & 66.85 & 970.12 & 10.30 & 3.28 & 6.64 \\
7.09 & 68.83 & 970.05 & 9.75 & 6.59 & 4.94 \\
9.89 & 70.55 & 969.97 & 9.33 & 8.33 & 15.62 \\
\hline
\end{tabular}

Table 4. The experimental data for $v=30.86 \mathrm{cSt}$.

\begin{tabular}{rrrrrr}
\hline $\mathrm{q}_{\mathrm{v} 0} / \mathrm{m}^{3} \mathrm{~h}$ & $\mathrm{t} /{ }^{\circ} \mathrm{C}$ & $\rho / \mathrm{kg} / \mathrm{m}^{3}$ & $\mathrm{v} / \mathrm{cSt}$ & $\mathrm{q}_{\mathrm{v}} / \mathrm{m}^{3} / \mathrm{h}$ & $\delta_{1} / \%$ \\
\hline 1.07 & 33.88 & 971.50 & 31.18 & 0.82 & 2.51 \\
2.52 & 33.98 & 971.50 & 31.08 & 2.06 & 4.59 \\
4.13 & 34.13 & 971.50 & 30.93 & 3.01 & 11.22 \\
7.05 & 34.35 & 971.48 & 30.65 & 5.25 & 18.01 \\
9.87 & 34.55 & 971.48 & 30.45 & 7.33 & 25.40 \\
\hline
\end{tabular}

Table 5. The experimental data for $v=38.13 \mathrm{cSt}$.

\begin{tabular}{rrrrrr}
\hline $\mathrm{q}_{\mathrm{v} 0} / \mathrm{m}^{3} / h$ & $\mathrm{t} /{ }^{\circ} \mathrm{C}$ & $\rho / k g / \mathrm{m}^{3}$ & $\mathrm{v} / \mathrm{cSt}$ & $\mathrm{q}_{\mathrm{v}} / \mathrm{m}^{3} / h$ & $\delta_{1} / \%$ \\
\hline 1.01 & 28.23 & 971.80 & 38.28 & 0.69 & 3.24 \\
2.55 & 28.48 & 971.78 & 37.85 & 2.00 & 5.45 \\
4.00 & 28.38 & 971.78 & 38.00 & 2.78 & 12.2 \\
7.01 & 28.18 & 971.78 & 38.35 & 4.86 & 21.45 \\
9.96 & 28.33 & 971.80 & 38.15 & 7.14 & 28.24 \\
\hline
\end{tabular}

Table 6. The experimental data for $v=50.96 \mathrm{cSt}$.

\begin{tabular}{rrrrrr}
\hline $\mathrm{q}_{\mathrm{v} 0} / \mathrm{m}^{3} / \mathrm{h}$ & $\mathrm{t} /{ }^{\circ} \mathrm{C}$ & $\rho / \mathrm{kg} / \mathrm{m}^{3}$ & $\mathrm{v} / \mathrm{cSt}$ & $\mathrm{q}_{\mathrm{v}} / \mathrm{m}^{3} / \mathrm{h}$ & $\delta_{1} / \%$ \\
\hline 1.05 & 22.00 & 972.10 & 52.53 & 0.63 & 4.19 \\
2.56 & 21.63 & 972.13 & 53.68 & 1.92 & 6.41 \\
4.01 & 22.08 & 972.10 & 52.28 & 2.68 & 13.36 \\
7.01 & 23.20 & 972.01 & 48.88 & 4.82 & 21.92 \\
9.91 & 23.70 & 972.00 & 47.43 & 6.47 & 34.47 \\
\hline
\end{tabular}

In the tables, $\mathrm{q}_{\mathrm{v} 0}$ is the rotameter indication flow rate, $\mathrm{q}_{\mathrm{v}}$ represents the actual flow rate of the 4050 oil obtained using the weighing method and $\delta_{1}$ is the full scale error:

$$
\delta_{1}=\frac{q_{v 0}-q_{v}}{q_{\max }} \times 100 \%
$$

As the lubricating oil density is close to the density of water, the medium density effects on the measurement can be ignored.

The experimental data indicate that at the same viscosity, the full scale error increases with the increase in flow rate. This is because with the increase in flow rate, the boundary layer thickness on the float and guide rod becomes thinner, making the velocity gradient in the boundary layer become larger. The viscous force on the float and guide rod becomes larger from the increasing velocity gradient in the boundary layer.

At the same indicated value, the full scale error becomes larger with the increase in medium viscosity. This is because the boundary layer thickness increases and the flow area is reduced as the viscosity increases. At the same time the viscous force is increased with the increase in medium viscosity. The full scale error therefore becomes larger due to the double resistance effect.

\section{Calculation Results AND ERROR ANAlysis}

Equation (40) is the flow rate formula considering the viscous friction. The calculated results according to (40) and the experimental error are shown in Fig.9. - Fig. 12.

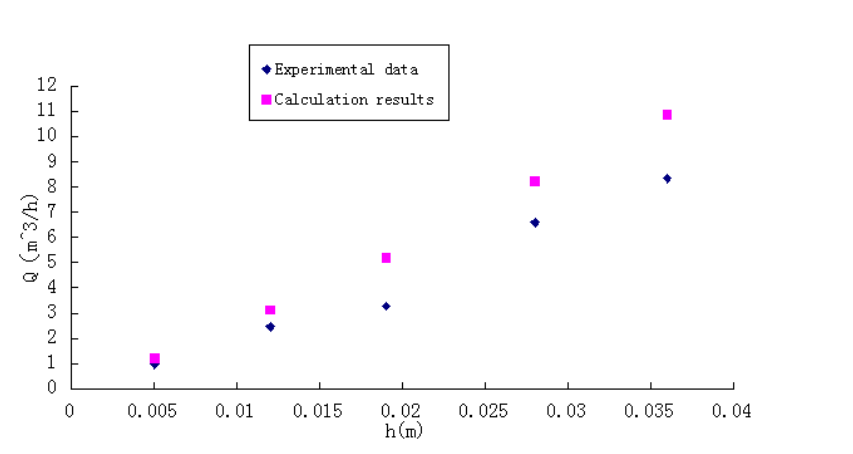

Fig.9. The calculated results for $\mathrm{v}=10 \mathrm{cSt}$.

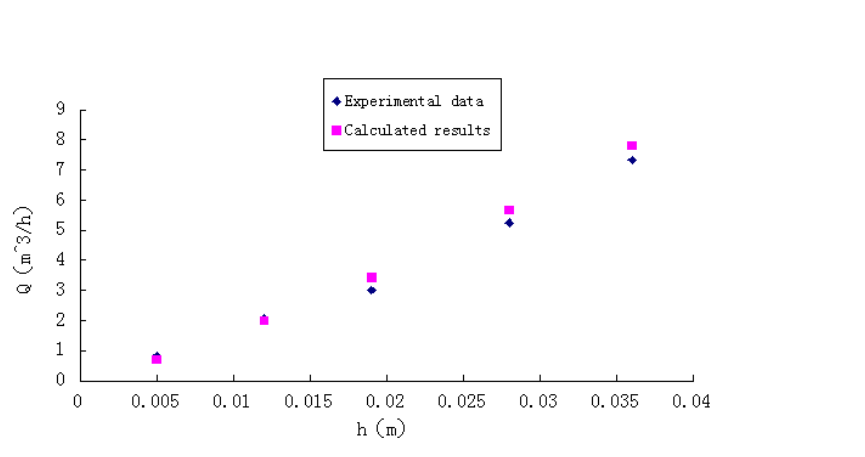

Fig.10. The calculated results for $\mathrm{v}=30 \mathrm{cSt}$. 


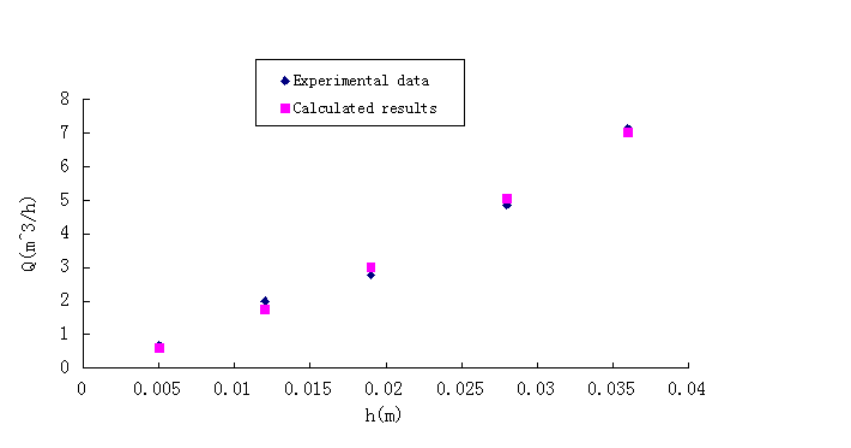

Fig.11. The calculated results for $v=40 \mathrm{cSt}$.

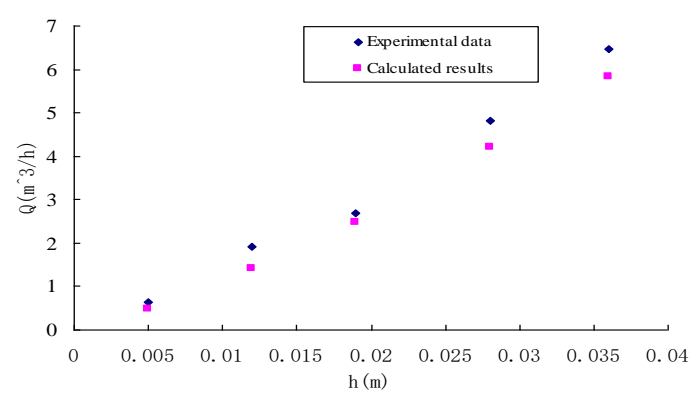

Fig.12. The calculated results for $\mathrm{v}=50 \mathrm{cSt}$.

Fig.9. demonstrates that at low viscosity all calculated results are larger than the experimental data. The error increases with the increase in flow rate. The calculation result shows that when $\mathrm{v}=10 \mathrm{cSt}$, the flow rate is $2.457 \mathrm{~m}^{3} / \mathrm{h}$, the rotameter Re number is 1259.4 at the annulus gap and the flow field in region $\mathrm{C}$ belongs to turbulence. When the flow field is in the turbulent state, the boundary layer separation phenomenon occurs, in which the boundary layer separates from the float surface and the recirculation zone will be produced at the largest float section and also at the corner of the orifice plate and pipe wall. The separation zone will seriously affect the boundary at the outflow region, which will change the wake meiobar range. The viscosity effect cannot be considered to only be limited to a thin layer of fluid near the surface. The pressure resistance calculation is not only related to the flow field distribution in region $\mathrm{C}$, but also in connection with the separation state and the wake region. Separation and turbulence will change the pressure difference spatial distribution greatly. There will be larger errors between the experimental data and calculated results based on the laminar flow model.

When $\mathrm{v}=30 \mathrm{cSt}$, the flow rate is $7.235 \mathrm{~m}^{3} / \mathrm{h}$ and $\mathrm{Re}$ is 1111.4. Fig.10. - Fig.12. illustrate that the error between the theoretical calculations and experimental data is very small, indicating that the model is reasonable.

\section{CONCLUSION}

The experimental and theory methods were used to examine the viscosity effect on rotameter measurement in this research. The experimental results indicate that at the same viscosity, the viscosity effect increases with the increase in flow rate. The error also increases at the same flow rate. The greater the viscosity becomes, the larger the error produced.
It is a new way that introduces wall jet and concentric annulus laminar theories to studying the influence of viscosity. A viscous fluid flow mathematical model through the orifice was established. And equation (40) is a new formula to calculate the flow rate at high viscosity. In the laminar flow the maximum error is only $6.3 \%$, indicating that the model has good laminar flow applicability.

In the turbulence state, because separation is produced at the corner of the orifice plate and the bottom and top of the float, the differential pressure spatial distribution consequently changes. Model calculation for differential pressure based on the laminar flow will generate a large error. On this occasion, relative to the viscous friction, the differential pressure plays a main role. The flow rate should be directly calculated using the classical rotameter equation.

\section{NOMENCLATURE}

$A_{1}=$ The cross-sectional area of concentric annular pipe after the outlet, $m^{2}$

$A_{f}=$ The area at the maximum cross section of float, $m^{2}$

$b_{0}=$ The width of outlet, $m$

$c_{1}, c_{2}, c_{3}, c_{4}=$ Constants associated with the structure of the float

$F_{\mathrm{p}}=$ The differential pressure force, $N$

$F_{\rho}=$ Buoyancy, $N$

$F_{\tau}=$ Viscous friction, $N$

$G=$ Gravity, N

$H=$ The length from float bottom to the top of bevel, $m$

$h=$ The distance of float moving in the current flow, $m$

$I_{1}(z), I_{2}(z)=$ Function associated with $\mathrm{z}$ coordinate

$J_{1}, J_{2}, J_{3}, J_{4}=$ Constants associated with the structure of the float

$K=$ Dimensionless coefficient characterization of the degree of limitation

$k=$ The radial length of front steps, $m$

$L=$ The distance from bottom of the float to the bottom of the orifice, $m$

$m=$ The position of outlet

$n=$ The position at the separation point of boundary layer

$\Delta p=$ Differential pressure, $P a$

$Q=$ Volume flow rate, $\mathrm{m}^{3} / \mathrm{h}$

$q_{\mathrm{v} 0}=$ Indication flow rate of the rotameter, $\mathrm{m}^{3} / \mathrm{h}$

$q_{\mathrm{v}}=$ The actual flow rate, $\mathrm{m}^{3} / \mathrm{h}$

$r_{1}=$ The tube radius, $m$

$r_{1}^{\prime}=$ The tube radius of region $\mathrm{B}_{1}, m$

$r_{2}=$ The float bottom radius, $m$

$r_{f}=$ Orifice radius, $m$

$S=$ The length of the cylinder at upper part of rotameter, $m$

$S_{A}, S_{B}, S_{B 1}, S_{B 2}=$ The annular area of the subscript, $m^{2}$

$u_{m}=$ The maximum velocity of the section in mixing zone, $\mathrm{m} / \mathrm{s}$

$u_{0}=$ Outlet velocity, $\mathrm{m} / \mathrm{s}$

$\alpha=$ Diffusion angle of the wall jet

$\beta=$ Float cone angle

$\theta=$ Rotation angle of column coordinates

$\varphi=$ Orifice cone angle

$\mu=$ Dynamic viscosity 


\section{REFERENCES}

[1] Head, V.P., Hatboro, P.A. (1954). Coefficients of float-type variable-area flowmeters. Transactions of the ASME, 76, 851-862.

[2] Schoenborn, E.M., Colburn, A.P. (1939). The flow mechanism and performance of the rotameter. Transactions of the American Institute of Chemical Engineers, 35, 359-389.

[3] Whitewell, J.C., Plumb, D.S. (1939). Correlation of rotameter flow rates. Industrial \& Engineering Chemistry, 31 (4), 451-456.

[4] Polentz, L.M. (1961). Theory and operation of rotameters. Instruments \& Control Systems, 34, 10481051.

[5] Urata, E. (1979). A new design of float-type variable area flowmeter. Bulletin of JSME, 22 (171), 12121219.

[6] Vallascas, R. (1987). New float flowmeter. Review of Scientific Instruments, 58 (8), 1499-1504.

[7] Liu, C.Y., Lua, A.C., Chan, W.K., Wong, Y.W. (1995). Theoretical and experimental investigations of a capacitance variable area flowmeter. Transactions of the Institute of Measurement and Control, 17 (2), 8489.

[8] Baker, R.C. (2004). The impact of component variation in the manufacturing process on variable area (VA) flowmeter performance. Flow Measurement and Instrumentation, 15 (4), 207-213.

[9] Baker, R.C., Sorbie, I. (2001). A review of the impact of component variation in the manufacturing process on variable area (VA) flowmeter performance. Flow Measurement and Instrumentation , 12 (2), 101-112.

[10] Sondh, H.S., Singh, S.N., Seshadri, V., Gandhi, B.K. (2002). Design and development of variable area orifice meter. Flow Measurement and Instrumentation, 13 (3) 69-73.

[11] Singh, S.N., Gandhi, B.K., Seshadri, V., Chauhan, V.S. (2004). Design of a bluff body for development of variable area orifice-meter. Flow measurement and Instrumentation, 15 (2), 97-103.

[12] Ning, J., Peng, J. (2009). A temperature compensation method based on neural net for metal tube rotameter. In International Conference on Transportation Engineering 2009. ASCE, 2334-2339.

[13] Bückle, U., Durst, F., Howe, B., Melling, A. (1992). Investigation of a floating element flowmeter. Flow Measurement and Instrumentation, 3 (4), 215-225.

[14] Bückle, U., Durst, F., Köchner, H., Melling, A. (1995). Further investigation of a floating element flowmeter. Flow Measurement and Instrumentation, 6 (1), 75-78.
[15] Turkowski, M. (2004). Influence of fluid properties on the characteristics of a mechanical oscillator flowmeter. Measurement, 35 (1), 11-18.

[16] Turkowski, M. (2003). Progress towards the optimization of a mechanical oscillator flowmeter. Flow Measurement and Instrumentation, 14 (1-2), 1321.

[17] Fisher, K. (1940). Elimination of viscosity as a factor in defining rotameter calibration. Transactions of the AIChE, 86, 857-869.

[18] Miller, R.W. (1983). Flow Measurement Engineering Handbook. McGraw-Hill, 1443-1458.

[19] Levin, H., Escorza, M.M. (1983). Gas flow through rotameters. Industrial \& Engineering Chemistry Fundamentals, 22 (2), 163-166.

[20] Wojtkowiak, J., Popiel, Cz.O. (1996). Viscosity correction factor for rotameter. Journal of Fluids Engineering, 118 (3), 569-573.

[21] Fredrickson, A.G. (1959). Flow of non-Newtonian fluids in annuli. Ph.D. University of Wisconsin.

[22] Glauert, M.B. (1956). The wall jet. Journal of Fluid Mechanics, 1 (6), 625-643.

[23] Launder, B.E., Rodi, W. (1979). The turbulent wall jet. Progress in Aerospace Sciences, 19, 81-128.

[24] van Hooff, T., Blocken, B., Defraeye, T., Carmeliet, J., van Heijst, G.J.F. (2012). PIV measurements of a plane wall jet in a confined space at transitional slot Reynolds numbers. Experiments in Fluids, 53 (2), 499517.

[25] Craft, T.J., Launder, B.E. (2001). On the spreading mechanism of the three-dimensional turbulent wall jet. Journal of Fluid Mechanics, 435, 305-326.

[26] Verhoff, A. (1963). The two-dimensional, turbulent wall jet with and without an external free stream. Report No. 626, Princeton University, NJ.

[27] Rajaratnam, N. (1967). Plane turbulent wall jets on rough boundaries. Water Power, 19, 149-153.

[28] Azim, M.A. (2013). On the structure of a plane turbulent wall jet. Journal of Fluids Engineering, 135 (8), 084502.

[29] Taliev, V.N. (1963). Ventilation Aerodynamics. Moscow: Gosstroiizdat, 1963.

Received November 11, 2015. Accepted April 05, 2016. 